\title{
Hepatitis C virus related cirrhosis: time to occurrence of hepatocellular carcinoma and death
}

\author{
F Degos, C Christidis, N Ganne-Carrie, J-P Farmachidi, C Degott, C Guettier, \\ J-C Trinchet, $M$ Beaugrand, S Chevret
}

\begin{abstract}
Background-In patients with hepatitis C virus (HCV) infection and cirrhosis, long term outcome and the incidence of hepatocellular carcinoma (HCC) are still debated.

Design-From January 1987 to January 1997, 416 patients (240 male, median age 57 years) with uncomplicated Child-Pugh A HCV related cirrhosis were followed in two Paris area centres from diagnosis of cirrhosis until death or reference date (1 June 1998). The analysis used a three state disability model generalising the Cox model.
\end{abstract}

Results-Of the 416 patients, 60 developed HCC with a five year rate of $13.4 \%(95 \%$ confidence interval (CI) 9.0-17.8\%) and 83 died (including 34 with HCC), with a five year death rate of $15.3 \%$ (95\% CI $12.6-$ $18.0 \%)$. By multivariable analysis, time to HCC relied on age (hazard ratio (HR) 1.05 per year; $p=0.0005)$, male sex (HR 2.13; $p=0.01$ ), oesophageal varices (HR 2.36; $p=$ 0.008), decreased platelet count (HR 0.99; $\mathrm{p}=0.03$ ), and bilirubin level (HR 1.01; $p=0.003$ ), while death after HCC was mainly related to tobacco consumption (HR 1.04; $p=0.0006)$. In contrast, death free of HCC was dependent on age (HR 1.04; $p=0.01$ ), oesophageal varices (HR 2.75; p=0.001), low platelet count (HR $0.99 ; p=0.006)$, and albumin level (HR $0.90 ; \mathrm{p}=0.0001$ ).

Conclusion-The incidence of HCC and mortality should be higher in these patients than previously stated, and prognostic factors of HCC and death are closely related age and symptoms of portal hypertension.

(Gut 2000;47:131-136)

Keywords: hepatitis C; cirrhosis; hepatocellular carcinoma; survival

Département de Biostatistique et Informatique

Médicale, Hôpital

Saint-Louis, Paris,

France

S Chevret

Correspondence to: F Degos, Service

d'Hépatogastroentérologie,

Hôpital Beaujon, 100

boulevard du général

Leclerc, 92110 Clichy,

France.

Email: francoise.degos@

bjn.ap-hop-paris.fr

Accepted for publication 8 February 2000

Hepatitis $\mathrm{C}$ virus (HCV) infection has become a major worldwide health problem because of the potential natural course of the disease to cirrhosis and then hepatocellular carcinoma (HCC).${ }^{1-14}$ At the present time, HCV related cirrhosis is the most common indication for countries $^{15}$ although limited data have been reported concerning long term mortality and incidence of HCC in these patients. ${ }^{512} 14$ Most previous retrospective cohort studies had several limitations caused by either the absence of HCV serology with focus on non-A non-B hepatitis $^{46710}$ or a short and incomplete follow-up. Prospective cohort studies used small samples with no consistent report of causes of death, ${ }^{16}$ except for a recent multicentre European study that enrolled, over a 10 year period, 384 patients with Child-Pugh A HCV related cirrhosis. ${ }^{17}$ Fair rates of survival of $91 \%$ and $79 \%$ at five and 10 years, respectively, were found. However, these estimates may have suffered from selection bias as some centres only included five patients.

Finally, the current understanding of prognostic factors for $\mathrm{HCV}$ related cirrhosis progression is based on consideration of only two states: the initial stage (cirrhosis) and an outcome stage (either HCC or death). Several authors were only interested in the occurrence of the outcome, ignoring the time scale and thus censoring. ${ }^{16}$ Others used time to outcome as the main end point, defining survival models. ${ }^{517-21}$ Almost all studies on prognostic factors have used this two state model as the basis for identifying progressive factors, with no details of interactions among the prognostic factors and outcome.

The aim of our study was to assess and predict the outcome of $\mathrm{HCV}$ related cirrhosis patients based on a cohort of 416 patients from two centres in the Paris area. Two main end points were considered, HCC and death, analysed jointly through the use of a three state disability model (or illness-death model). ${ }^{22}$

\section{Patients and methods}

PATIENTS

Inclusion in the study was considered at the time of diagnosis of $\mathrm{HCV}$ related cirrhosis. All consecutive patients with HCV related cirrhosis referred to our two hepatology departments between 1 January 1987 and 31 December 1996 for liver biopsy were included in the study if they met all of the following criteria: (1) positive serum anti-HCV antibodies (second or third generation ELISA test); (2) cirrhosis compatible with HCV origin proved on biopsy either by the transparietal or transvenous route; orthotopic liver transplantation in Western
Abbreviations used in this paper: $\mathrm{HCV}$, hepatitis $\mathrm{C}$ virus; HCC, hepatocellular carcinoma; AFP, $\alpha$ fetoprotein; SMR, standardised mortality ratio; HR, hazard ratio. 
Table 1 Main characteristics of the 416 patients at the time of $\mathrm{HCV}$ related cirrhosis

\begin{tabular}{ll}
\hline Age (y) & $57(46-64)$ \\
Male sex & $240(58 \%)$ \\
Daily alcohol consumption (g) & \\
$\quad<40$ & $334(83 \%)$ \\
$40-80$ & $28(7 \%)$ \\
$\quad \geqslant 80$ & $40(10 \%)$ \\
Smokers & $117(28 \%)$ \\
Tobacco consumption (pack-year) & $0(0-15)$ \\
Contamination: blood transfusion & $196(47 \%)$ \\
Oesophageal varices & $175(42 \%)$ \\
$\quad$ Grade $1 / 2 / 3$ & $127 / 47 / 8$ \\
Ascitis & $45(11.7 \%)$ \\
Albumin level (g/l) & $41(38-45)$ \\
Bilirubin level ( $\mu$ mol) & $15(11-21)$ \\
Patients with bilirubin $>30 \mu$ mol & $38(9.7 \%)$ \\
Platelet count $\left(10^{9} / 1\right)$ & $123(88-170)$ \\
Prothrombin time $(\%)$ & $78(68-92)$ \\
$\alpha$ Fetoprotein $(\mathrm{ng} / \mathrm{ml})$ & $10(5-20)$ \\
Knodell activity score & $7(6-11)$ \\
$\quad$ Minimal $<4$ & $206(50 \%)$ \\
$\quad$ Moderate $4-8$ & $169(41 \%)$ \\
Severe $>8$ & $41(9 \%)$ \\
Large cell dysplasia $(287$ patients) & $44(15 \%)$ \\
Interferon therapy & $223(54 \%)$ \\
\hline
\end{tabular}

Values are median (25-75\% quantiles) or number (\%).

France allowing regular follow up. The date of the liver biopsy establishing the diagnosis of cirrhosis defined the starting time in the study.

BASELINE CLINICAL AND BIOLOGICAL DATA

Routine clinical and biological data were collected: age, sex, tobacco consumption, serum albumin $(\mathrm{g} / \mathrm{l})$, bilirubin $(\mu \mathrm{mol} / \mathrm{l})$, platelet count $\left(10^{9} / 1\right)$, prothrombin time $(\%)$, and AFP level $(\mathrm{ng} / \mathrm{ml})$. Daily alcohol consumption was evaluated as < 40 g, 40-80 g, and > $80 \mathrm{~g}$. Oesophageal varices were looked for at endoscopy in 325 patients (table 1 ).

LIVER HISTOLOGY

All patients underwent transparietal or transvenous liver biopsy depending mainly on platelet count (with a cut off of $100 \times 10^{9} / 1$ ) and bleeding time. Transvenous biopsies were also performed for protracted prothrombin times or for the purpose of measuring wedged hepatic pressure. Liver biopsy samples were fixed and embedded in paraffin. Knodell fibrosis and activity scores were assessed. ${ }^{23}$ Of the 416 biopsies, 278 were independently re-examined by two liver pathologists (CD and $C G$ ), without knowledge of the clinical data, to detect the presence of large cell dysplasia. Small liver samples obtained from transvenous liver biopsies were not analysed for the presence of large cell dysplasia. Nonconcordant results involved reviewing the slides until consensus was established.

PROSPECTIVE FOLLOW UP AND HCC SCREENING In accordance with the screening programme, ultrasonographic study of the liver was performed every six months. A diagnosis of HCC was based on histology obtained by ultrasound guided liver biopsy. In patients in whom liver biopsy was not possible, a diagnosis was based on: (a) liver cirrhosis; (b) focal lesion compatible with HCC, demonstrated by two imaging techniques (ultrasound, CT scan, or MRI); and (c) AFP level above $400 \mathrm{ng} / \mathrm{ml}$, portal thrombosis, or growing arterial hypervascular tumour at CT scan and/or angiography and/or

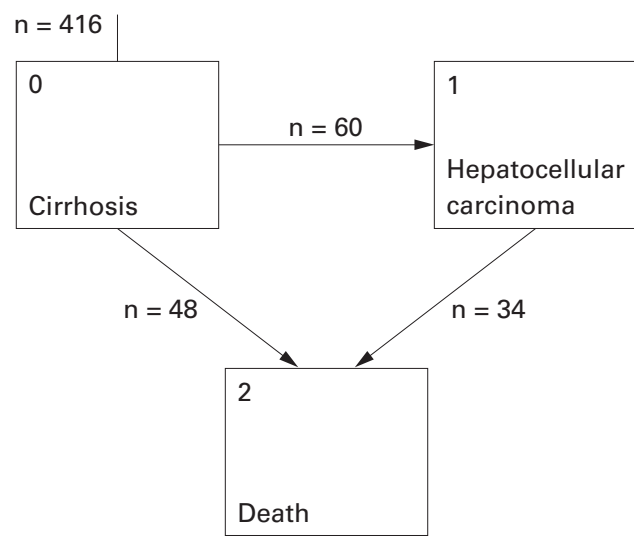

Figure 1 Three state disability model. Each box refers to one state (cirrhosis, hepatocellular carcinoma, or death), while arrows denote possible transitions between these states.

MRI. Doubtful cases were not considered as HCC until a precise histological diagnosis was obtained. All patients considered as HCC without histological proof were followed up and had an increase in the size of their tumour.

INTERFERON TREATMENT

Interferon treatment was recorded. Sustained response was defined on biological criteria (normalisation of alanine aminotransferase activity six months after discontinuation of treatment). Because of the length of the study, we did not evaluate the virological response to treatment.

\section{STATISTICAL ANALYSIS}

Analysis was based on the reference date of 1 June 1998. The disability model was specified by three transition intensities: the intensity (or incidence) of developing HCC, the incidence of death without HCC, and the incidence of death intensity with HCC (fig 1). Analysis of the disability model is performed one transition at a time, using time since diagnosis of $\mathrm{HCV}$ related cirrhosis as the time scale. ${ }^{24}$ Patients were at risk of death unrelated to HCC from a diagnosis of cirrhosis until the time to death without HCC, censoring, or development of HCC. They were at risk of death related to HCC from the time to development of HCC until the time to death or censoring. We first estimated time to events using the Kaplan Meier method, and the hazard rate over one year intervals by the actuarial life table method. Standardised mortality ratios (SMR) were computed to compare survival distributions with those of the general French population, matched for age and sex.

Prognostic analyses considered for each transition intensity a full multivariable model with proportional hazards that allowed estimation of the hazard ratio (HR) with 95\% confidence intervals (95\% CI). The fit of the final model was checked by testing for interaction between the prognostic factors in the model and the underlying time (that is, for proportional intensities) and by testing the log linearity of the continuous predictors. The latter were also introduced as binary covariates after dichotomisation according to median values. 

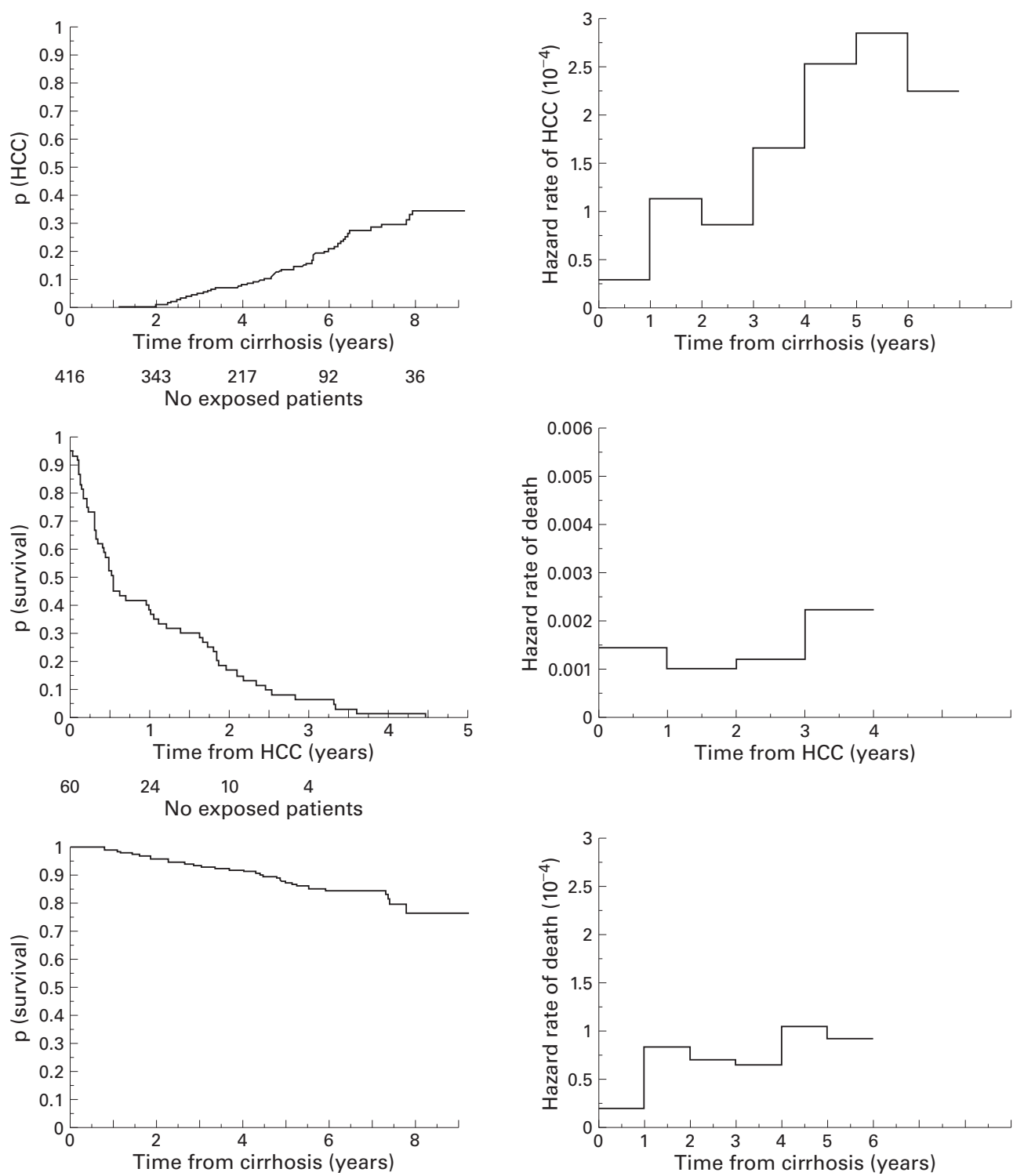

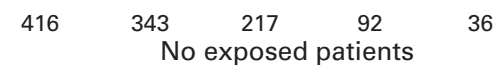

Figure 2 Estimated time to outcome (hepatocellular carcinoma (HCC), death after HCC, or death free of HCC) with associated estimated hazard of developing the event over one year time intervals. This refers to the conditional failure rate (that is, the probability of an individual free of an event at the end of the time interval experiencing the event in the next interval). An interesting feature of these data is that the hazard rate for HCC increases over time while the hazard rates for death remain roughly constant over time.

Two sided tests were computed with $\mathrm{p}$ values $<0.05$ defining statistical significance. SAS (SAS Inc, Cary, North Carolina, USA) and S-plus software packages were used for statistical analysis.

\section{Results}

BASELINE CHARACTERISTICS

From 1 January 1989 to 31 December 1996, 651 consecutive patients with Child A HCV cirrhosis were referred to our two departments for liver biopsy. Of these, 215 were excluded because of viral coinfection, the presence of an ultrasound detectable nodule at inclusion, and/or non-virus $C$ related liver disease. Accordingly, 416 patients fulfilled the inclusion criteria. A liver biopsy was performed by the transvenous transjugular route in $58 \%$ of patients. Haemodynamic measurements were not available for all patients.

The sample included 240 males and 176 females, median age 57 years. Table 1 summarises the distribution of the baseline patient characteristics at the time of diagnosis of $\mathrm{HCV}$ related cirrhosis. The majority of patients $(80 \%)$ were asymptomatic, the diagnosis of cirrhosis being the result of screening liver biopsy, while $20 \%$ were symptomatic: 45 patients $(11.7 \%)$ had minimal US detectable ascitis and bilirubin was $>30<50 \mu \mathrm{mol} / 1$ in 38 patients $(9.7 \%)$. According to the Knodell score, all 416 patients had a fibrosis index of 4 while the activity index was minimal $(<4)$ in $206(50 \%)$ patients, moderate (4-8) in $169(41 \%)$, and severe (>8) in $41(9 \%)$. Large cell dysplasia was observed in $44 / 287$ patients $(15 \%)$. 

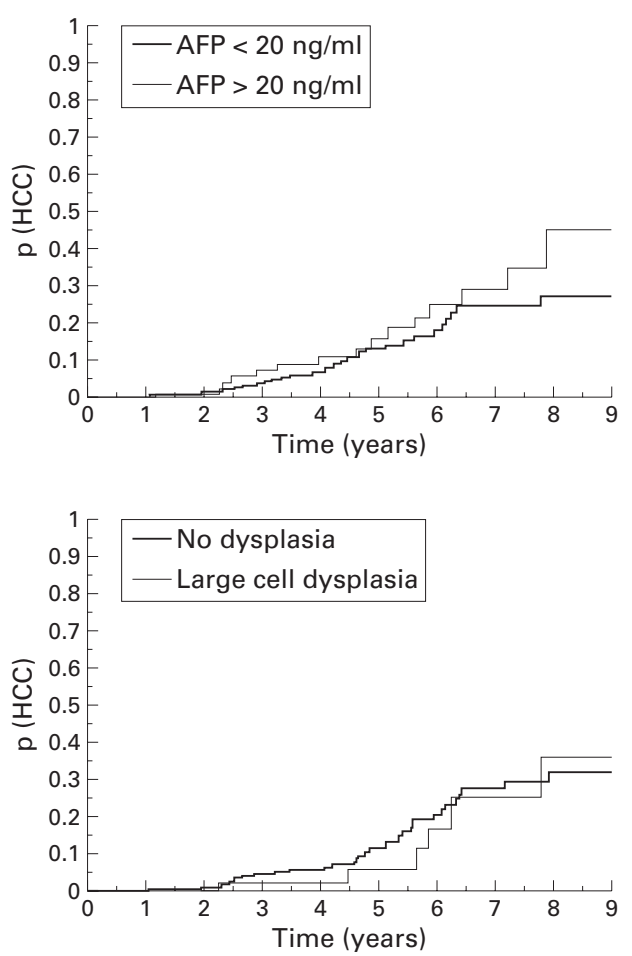

Figure 3 Estimated time to hepatocellular carcinoma (HCC) according to serum a fetoprotein (AFP) levels ( \pm $20 \mathrm{ng} / \mathrm{ml}$ ) and large cell dysplasia.

Interferon therapy was instituted in 223 patients on the physician's decision in the absence of prospective predetermined criteria. Alpha interferon was administered subcutaneously at a daily dose of 3 MUI TTW for six months to 126 patients while the dose or duration of treatment was reduced mainly because of haematological disorders (thromboleucopenia) or fatigue in 97 patients; a sustained biochemical response was observed in 38 patients $(17 \%)$. Other treatments, namely treatment of portal hypertension with $\beta$ blockers, sclerotherapy, and band ligation, were not recorded. Median duration of follow up was 68 months (4-199); the mean interval of time between two ultrasound liver examinations was 8.2 months.

INCIDENCE OF HCC AND MORTALITY

At the reference date, 60 hepatocellular carcinomas and 83 deaths were observed, including 34 after HCC (fig 2). Among patients with HCC, the tumour was unique in $62 \%$ and multiple at the first investigation in
$37 \%$. The size of the confirmed HCC tumour was $>10 \mathrm{~mm}$ in all but two patients. AFP level was $>400 \mathrm{ng} / \mathrm{ml}$ in $17 \%$ of patients (fig 3). At five years the HCC rate was $13.4 \%$ (95\% confidence interval (CI) $9.0-17.8 \%$ ) and the death rate was $15.25 \%$ (95\% CI 12.6-17.9\%). Death was not due to liver disease in 19 patients (including suicide in one untreated patient): cancer (ovarian, digestive, brain tumours) $(n=6)$; cardiac failure $(n=4)$; severe infection $(n=3)$; brain haemorrhage $(n=2)$; other $(n=3)$. Compared with age and sex matched life tables, mortality for $\mathrm{HCV}$ related cirrhosis whatever the cause was increased by about threefold (SMR 2.85; p<0.0001). Analysing only mortality of $\mathrm{HCV}$ related cirrhosis (that is, excluding HCC related mortality) yielded an estimated SMR of $1.76(p=0.001)$. Interestingly, the hazard rate of HCC tended to increase with time, when the hazard rates for death remained fairly constant (fig 2).

\section{PROGNOSTIC ANALYSES}

The results of the prognostic multivariate analyses are summarised in table 2 . The occurrence of HCC was related to increased age (hazard ratio (HR) 3.78 above 57 years; 95\% CI 1.77-8.05), male sex (HR 2.13; 95\% CI 1.18-3.85), presence of oesophageal varices (HR 2.36; 95\% CI 1.26-4.45), decreased platelet count (HR 1.69 below $100 \times 10^{9} / 1 ; 95 \%$ CI 0.99-3.17), and bilirubin level (HR 1.14 above $15 \mathrm{mg} / \mathrm{dl} ; 95 \%$ CI 0.61-2.13), all assessed at the time of diagnosis of cirrhosis. Alcohol consumption was not significantly associated with the occurrence of HCC.

To explain the heterogeneity in survival times without HCC, the most relevant variables were close to those related to the occurrence of HCC, suggesting a homogeneous baseline high risk group based on age (HR 1.31 ; 95\% CI 0.63-2.72), presence of oesophageal varices (HR 2.75; 95\% CI 1.48-5.10), decreased albumin level (HR 3.70 below $41 \mathrm{~g} / 1$; 95\% CI 1.57-8.70), and platelet count (HR 1.37 below $100 \times 10^{9} / 1$; $95 \%$ CI $0.66-2.84$ ).

\section{Discussion}

This series based on 416 Child A patients with HCV related cirrhosis has allowed a better description and understanding of the course of the disease in terms of occurrence of either HCC or death.

In contrast with the previous large Eurohep study, ${ }^{17}$ mortality was higher in our series (with

Table 2 Prognosis analyses according to outcome: hepatocellular carcinoma (HCC), death after hepatocellular carcinoma, and death unrelated to hepatocellular carcinoma

\begin{tabular}{|c|c|c|c|c|c|c|c|c|c|}
\hline & \multicolumn{3}{|c|}{ HCC (60 events) } & \multicolumn{3}{|c|}{ Death after HCC (34 events) } & \multicolumn{3}{|c|}{ Death unrelated to HCC (48 events) } \\
\hline & $H R$ & $(95 C I)$ & $p$ Value & $\overline{H R}$ & $(95 C I)$ & $p$ Value & $\overline{H R}$ & $(95 C I)$ & $p$ Value \\
\hline Age & 1.05 & $(1.02-1.08)$ & 0.0005 & 1.03 & $(0.98-1.08)$ & 0.23 & 1.04 & $(1.01-1.07)$ & 0.014 \\
\hline Female & 0.47 & $(0.26-0.85)$ & 0.013 & 0.96 & $(0.38-2.40)$ & 0.93 & 1.12 & $(0.61-2.09)$ & 0.71 \\
\hline Oesophaeal varices* & 2.36 & $(1.25-4.45)$ & 0.008 & 0.63 & $(0.26-1.53)$ & 0.30 & 2.75 & $(1.48-5.10)$ & 0.0013 \\
\hline Platelets & 0.99 & $(0.98-0.99)$ & 0.029 & 1.002 & $(0.99-1.01)$ & 0.61 & 0.99 & $(0.98-0.99)$ & 0.006 \\
\hline Bilirubin & 1.01 & $(1.005-1.02)$ & 0.003 & 1.000 & $(0.99-1.01)$ & 0.98 & 1.003 & $(0.99-1.01)$ & 0.61 \\
\hline Alcohol > $40 \mathrm{~g}$ & 0.56 & $(0.26-1.23)$ & 0.15 & 0.45 & $(0.14-1.46)$ & 0.18 & 0.94 & $(0.41-2.17)$ & 0.89 \\
\hline Tobacco & 1.01 & $(0.99-1.02)$ & 0.42 & 1.04 & $(1.02-1.06)$ & 0.0006 & 1.005 & $(0.99-1.02)$ & 0.56 \\
\hline Albumin & 1.03 & $(0.97-1.09)$ & 0.36 & 0.93 & $(0.86-1.01)$ & 0.07 & 0.90 & $(0.86-0.95)$ & 0.0001 \\
\hline
\end{tabular}

${ }^{\star}$ Grade 2 or $>2$

HR, hazard ratio; $95 \mathrm{CI}, 95 \%$ confidence interval. 
for example a five year death rate of $15 \%$ compared with $9 \%$ ) although all patients had a similar inclusion criterion (that is, Child-Pugh class A). This could be explained by the extensive use of transvenous liver biopsy in our study, allowing us to include patients with more severe liver disease. Moreover, inclusion in our study, contrary to the Eurohep study, ${ }^{17}$ of $10 \%$ of patients with a large (>80 g/day) alcohol consumption (provided that histological examination showed a pattern of $\mathrm{HCV}$ related disease) may have contributed to our overall high death rate. However, most of these patients stopped or reduced their drinking habits during follow up (evaluation based on questioning by the physician) and alcohol consumption at the initiation of follow up was not found to be a prognostic factor in our study.

With regard to the occurrence of HCC, we first showed that the instantaneous risk of HCC increased with each passing year, as shown by Mazella and colleagues. ${ }^{20}$ This could be explained in part by exclusion of cirrhotic patients with a non-identified, even small liver nodule at entry, as well as patients with AFP $>50 \mathrm{ng} / \mathrm{ml}$. Moreover, it could be related to increasing risk factors such as more advanced age and poorer liver function. Overall, the incidence of HCC was high, with an estimate of $13.4 \%$ at five years compared with previous reports of $7 \%{ }^{17}$ or $5 \% .^{20}$ Such a discrepancy could be due to improvements in the accuracy of diagnosis and closer follow up for early detection of HCC. Nevertheless, our estimate is in the range of previous reports using a similar mean time and periodicity of follow up, and showing an incidence of $11 \%$ at four years ${ }^{16}$ and $13.5 \%$ at five years. ${ }^{25}$

In understanding the variability of the occurrence of HCC in HCV related cirrhosis patients, we confirmed the importance of sex, age, and signs of portal hypertension, such as oesophageal varices and low platelet count, as previously published. ${ }^{19}{ }^{20}$ The prognostic value of haemodynamic measures and the correlation with platelet count require further study. We did not find any relationship between the occurrence of HCC and previously reported prognostic factors such as alcohol consumption and cigarette smoking, ${ }^{14} 20$ but, as discussed below, surprisingly cigarette smoking was a predictive factor of death in patients with HCC.

Levels of AFP at inclusion did not add any prognostic information on HCC occurrence in contrast with a previous report. ${ }^{14}$ This relates to AFP levels obtained at the start point of the study and below $50 \mathrm{ng} / \mathrm{ml}$. It does not rule out a prognostic value of serum levels of AFP above $50 \mathrm{ng} / \mathrm{ml}$ or of a transient elevation during the follow up, as only the initial value was taken into account. More interestingly, in our study, two other controversial predictive factors were not found to be associated with the occurrence of HCC, namely the presence of large cell dysplasia and treatment with interferon.

The liver biopsies at inclusion of 278/416 patients were reviewed independently by two pathologists (CD and CG), blinded to the outcome, so that there would be no bias in the results. The predictive value of large cell dysplasia may depend on the cause of cirrhosis as previous findings were based on patients with heterogeneous causes (alcohol, hepatitis B virus infection, hepatitis $C)^{25} 26$ and not specifically on HCV. In fact, large cell dysplasia is probably more informative of outcome in HBV infection $^{26}$ or even alcoholic cirrhosis which largely predominates in some studies. ${ }^{27}$

The role of interferon in the prevention of HCC has been widely discussed, ${ }^{16-18} 2021$ 28-32 and our results only underline the need for a large prospective randomised trial. No conclusion can be drawn from the absence of significant results in our study. Finally, virological factors such as the presence of HBV markers ${ }^{28}$ of $\mathrm{HCV}$ replication detected by $\mathrm{PCR}^{8}$ and infection with genotype $1^{19}{ }^{32}$ that have been described as predictive of HCC could not be assessed in our patients. HBV markers have been previously studied in patients from one centre (Bondy) and did not seem to add any prognostic value to the occurrence of HCC.

In the small group of 34 patients with HCC, the occurrence of death was independently related to smoking habits. The importance of tobacco in hepatocarcinogenesis has been stressed by previous studies. Tobacco smoking, especially when associated with alcohol consumption, could induce metabolites that have a carcinogenic effect, and presumably for explaining our data could lead to more malignant forms of HCC.

In our series, the predictive factors of survival in patients free of HCC were similar to those found in the Eurohep study, ${ }^{17}$ namely age, low platelet count, and high bilirubin level at initial examination. We also found that decreased albumin level and the presence of oesophageal varices were predictive of poor survival. The various treatments of portal hypertension were not recorded and their influence on mortality was therefore not studied. In fact, these factors together reflect the importance of age on the severity of liver disease in predicting outcome unrelated to HCC.

It should be noted that the data requirements for a multi-state model are quite strict. Not only are the dates of entry and death or censoring needed, but also, in principle, the exact transition times between the transient states must be known for each individual. In our data set, patients with HCV related cirrhosis underwent a screening programme that allowed for similar detection of HCC. Nevertheless, it should be kept in mind that similar problems may arise with any clinical data when the detection of non-fatal complications depends strongly on the frequency with which patients are followed. Finally, the value of any set of prognostic factors depends on reproducibility in a new patient sample. Our results agree with previously published data and appear to confirm the stability of the predictive factors that were identified. These factors could be of great importance in selecting high risk patients for intensive follow up and early detection of HCC. 
The study was supported by a grant from the Association de Recherche contre le Cancer.

1 Alter MJ, Margolis HS, Krawczynski K, et al. The natural history of community-acquired hepatitis $\mathrm{C}$ in the United history of community-acquired hepatitis
States. N Engl f Med 1992;327:1899-905.

2 Arista-Nasr J, Pichardo-Bahena R, Castaneda B, et al. Hepatitis C: a disease with a wide morphological spectrum? f Clin Gastroenterol 1996;22:121-5.

3 Caselmann WH, Alt M. Hepatitis C virus infection as a major risk factor for hepatocellular carcinoma. $\mathcal{F}$ Hepatol 1996;24S:61-6.

4 Di Bisceglie AM, Goodman ZD, Ishak KG, et al. Long-term clinical and histopathological follow-up of chronic posttransfusion hepatitis. Hepatology 1991;14:969-74.

5 Ikeda K, Saitoh S, Koida I, et al. A multivariate analysis of risk factors for hepatocellular carcinogenesis: a prospective observation of 795 patients with viral and alcoholic cirrhosis. Hepatology 1993;18:47-53.

6 Kiyosawa K, Sodeyama T, Tanaka E, et al. Interrelationship of blood transfusion, non-A, non-B hepatitis and hepatocellular carcinoma: analysis by detection of antibody to hepatitis C virus. Hepatology 1990;12:671-5.

7 Koretz RL, Abbey H, Coleman E, et al. Non-A, non-B postKoretz RL, Abbey H, Coleman E, et al. Non-A, non-B post-
transfusion hepatitis. Ann Intern Med 1993;119:110-15.

8 Naoumov NV, Chokshi S, Metivier E, et al. Hepatitis C virus infection in the development of hepatocellular carcinoma infection in the development of hepa
in cirrhosis. $\mathcal{F}$ Hepatol 1997;27:331-6.

9 Roudot-Thoraval F, Bastié A, Pawlotsky JM, et al. Epidemiological factors affecting the severity of hepatitis $C$ virus-related liver disease: a French survey of 6,664 patients. Hepatology 1997;26:485-90.

10 Seeff LB, Buskell-Bales Z, Wright EC, et al. Long-term mortality after transfusion-associated non-A, non-B hepatitis. N Engl F Med 1992;327:1906-11.

11 Simonetti RG, Camma C, Fiorello F, et al. Hepatitis C virus infection as a risk factor for hepatocellular carcinoma in patients with cirrhosis. A case-control study. Ann Intern Med 1992;116:97-102.

12 Takano S, Yokosuka O, Imazeki F, et al. Incidence of hepatocellular carcinoma in chronic hepatitis $\mathrm{B}$ and $\mathrm{C}$ : a prospective study of 251 patients. Hepatology 1995;21:65055 .

13 Tong MJ, El-Farra NS, Reikes A, et al. Clinical outcomes after transfusion-associated hepatitis C. $N$ Engl $7 \mathrm{Med}$ after transfusion-

14 Tsukuma H, Hiyama T, Tanaka S, et al. Risk factors for hepatocellular carcinoma among patients with chronic live hepatocellular carcinoma among patients with
disease. N Engl f Med 1993;328:1791-801.

15 Terrault NA, Wright TL. Hepatitis C virus in the setting of transplantation. Semin Liver Dis 1995; 15:92-100.

6 Serfaty L, Aumaître $\mathrm{H}$, Chazouillières $\mathrm{O}$, et al. Determinant of outcome of compensated hepatitis $\mathrm{C}$ virus-related cirrhosis. Hepatology 1998;27:1435-40

17 Fattovich G, Giustina G, Degos F, et al. Morbidity and mortality in compensated cirrhosis type C: a retrospective follow-up study of 384 patients. Gastroenterology 1997;112 463-72.
18 Imai Y, Kawata S, Tamura S, et al. Relation of interferon herapy and hepatocellular carcinoma in patients with chronic hepatitis C. Ann Intern Med 1998;129:94-9.

19 Kasahara A, Hayashi N, Mochizuki K, et al. Risk factors for hepatocellular carcinoma and its incidence after interferon treatment in patients with chronic hepatitis C. Hepatology 1998;27:1394-402.

20 Mazzella G, Accogli E, Sottili S, et al. Alpha interferon treatment may prevent hepatocellular carcinoma in HCVrelated liver cirrhosis. 7 Hepatol 1996;24:141-7.

21 Nishiguchi S, Kuroki T, Nakatani S, et al. Randomised trial of effects of interferon alpha on incidence of hepatocellular carcinoma in chronic active hepatitis C with cirrhosis. Lancet 1995;346:1051-5.

22 Andersen PK. Multistate models in survival analysis: a study of nephropathy and mortality in diabetes. Stat Med 1988;7: 661-70.

23 Knodell RG, Ishak KG, Black WC, et al. Formulation and application of a numerical scoring system for assessing hisapplication of a numerical scoring system for assessing hisHepatology 1981;1:431-5.

24 Andersen PK, Borgan Ø. Counting process models for life history data: a review (with discussion). Scand F Stat 1985; 12:97-158.

25 Ganne-Carrie N, Chastang C, Chapel F, et al. Predictive score for the development of hepatocellular carcinoma and additional value of large cell dysplasia in western patients with cirrhosis. Hepatology 1996;23:1112-18.

26 Borzio M, Bruno S, Roncalli M, et al. Liver cell dysplasia is a major risk factor for hepatocellular carcinoma in cirrhosis: a prospective study. Gastroenterology 1995;108: $812-17$

27 Ganne-Carrie N, Chastang C, Pateron D, et al. Does a previous infection by hepatitis $\mathrm{B}$ virus play a role in the occurrence of hepatocellular carcinoma in western patients with cirrhosis mainly due to alcohol or hepatitis $\mathrm{C}$ virus? Hepatology 1996;24S:1374A.

28 International Interferon Alpha Hepatocellular Study Group. Effect of interferon alfa on progression of cirrhosis to hepatocellular carcinoma: a retrospective cohort study. Lancet 1998:351:1535-9.

29 Shiratori Y, Yokosuka R, Ihori M, et al. Prospective study of interferon therapy for compensated cirrhotic patients with chronic hepatitis $\mathrm{C}$ by monitoring serum hepatitis C RNA. Hepatology 1999;29:1573-80.

30 Valla DC, Chevallier M, Marcellin P, et al. Treatment of hepatitis $\mathrm{C}$ virus related cirrhosis: a randomized controlled trial of interferon alfa $2 \mathrm{~b}$ versus no treatment. Hepatology 1999;29:1870-5.

31 Fattovich G, Giustina G, Degos F, et al. Effectiveness of interferon alfa on incidence of hepatocellular carcinoma and decompensation in cirrhosis type C. $\mathcal{f}$ Hepatol 1997;27:201-5.

32 Bruno S, Silini E, Crosignani A, et al. Hepatitis C virus genotypes and risk of hepatocellular carcinoma in cirrhosis: a prospective study. Hepatology 1997;25:754-8. 